nebensächliche sein kann;" und weiter: ,und dië Ver. sucbe von Molliard ergaben, dab grüne Pflanzen Humus-C für sich verwenden können." In der angezogenen Originalarbeit ${ }^{1}$ ) steht das genaue Gegenteil aer von Czapek geschriebenen Sätze, nämlich, daB die aus $\mathrm{Hu}$ mus entbundene Fohlensiiure einen wesentlichen Einfhu auf die Vegetation ausübt, und daB Humussubstanzen, wenn uberhaupt, so doch nur in gering fügigem Maße von den grünen Pflanzen direkt, also durch die Wurzeln aufgenommen werden. Wörtlich lauten die Sätze ,Ces premières expériences mettent en évidence l'action que l'humus peut exercer sur la vegé tation par sa production d'anbydride carbonique; je montrerai ailleurs que certains caractères de morphologie extérieure et d'anatomie des plantes cultivês en châssis et sur terreau s'expliquent par cela mềme . maís ces premiers résultats permettent déjà de conclure que, si des matières humiques sont assimilées directement par les plantes vertes, ce n'est que d'une manière tout a fait insignifiantec6.

Bemerkenswert ist, daA die Ergebnisse der Untersuchungen Molliards in der zweiten Auflage der „Biochemie der Pflanzen" von Fr. Czapek, S. 499, richtig wiedergegeben sind.

Heidelberg, den 3. Mai 1920

Prof. Dr. Borncmann.

\section{Zur Theorie der Zwischenstufen bei} chemischen Umwandlungen ${ }^{2}$ ).

In der geschichtlich so bedeutsamen Abhandlung August Kelculés ,Uber die Konstitution und die Metamorphosen der cbëmischen Verbindungen und uber die chemische Natur des Kohlenstofis" in Ann. d. Chemie 106,129-159 (1858), findet sich gelegentlich der ,auf alle chemische Metamorphosen anwendbaren Vorstellung" folgende Anmerkung (S. 141)

,Man kann sich denken, daß dabei während der Annäherung der Moleküle schon der Zusammenhang der Atome in denselben gelockert wird, weil ein Teil ${ }^{3}$ ) der Verwandtschaftskraft durch die Atome des anderen Moleküls gebunden wird, bis endlich die vorher vereinigten Atome ganz ihren Zusammenhang verlieren. und die neu gebikdeten Moleküle sich trennen . . Massenwirkung und Katalyse unterscheiden sich dieser Auffassung nach nu.r dadurch voneinander, daß bei Massenwirkung das katalytisch-wirkende Molekül gleichartig mit einem der sich zersetzenden, bei Katalyse dagegen stofflich verschieden von beiden ist."

C. F. Schönbein hatte vorher (Brief an Liebig vom 5. IX. 1853) ähnliche Gedanken, allerdings nicht auf der von ihm wenig geschätzten atomistischen, sordern auf "dynamischer"* Grundlage angedeutet.

Mit diesem Hinweise auf so weit zurückliegende AuBerungen soll natürlich nicht ihre Identität mit der neuen Theorie behauptet werden. Aber es ist doch interessant, dem gleichen Grundgedanken auch dort zu begegnen und dann zu sehen, wie weit er heute als verwirklicht und in Einzelheiten bestimmt gelten kann. Dazwischen lag die Anschauung von der Unteilba'rkeit und Komstanz der Valenzen. Gerade weil sie zu ihrer Zeit einen wertvollen Fortschritt bedeutete, erkennt man auch an diesem Falle einen typischen Verlauf solcher Entwicklungen.

Berlin-Dahlem, den 1. Mai 1920.

Eduard F'ärber.

1) Molliard, Compt. rend. T. 154, S. 291 (1912).

2) Vgl. Die Naturwissensehaften $8,322,1920$.

3) Von mir gesperrt.
Zur Kenntnis des grïnen Strahls.

$Z u$ der Mitteilung von $B$. Brandt über den grünen Strahl sind folgende Ergänzungen wohl von Interesse. Die Erscheinung ist auch bei uns zu beobachten. Ich sah sie zweimal. Zum erstenmal vor etwa 13 Jahren bei Göttingen und zum zweitenmal in diesem Frühling bei Bonn. Es war ein Abend von ganz ungewöhnlicher Klarheit. Die Beobachtung drängte sich ohne daß an sie gedacht war - auf und konnte von meinem Begleiter, dem die Erscheinung unbekannt war, sofort bestätigt werden. Die Farbe der Lichtenscheinung war hellsmaragdgrün und hatte etwa die Gestalt eines Halbkreises über der Stele, an der eben die Sonne verschwunden war. Die Dauer betrug einige Sekunden. Auch in A.gypten ist der grüne Strahl durchaus nicht regelmïBig zu sehen, wenn auch wohl häufiger als bei uns. Genaue Beobachtungen darüber teilt der Agyptologe Groff') mit, aus denen folgendes bemerkenswert ist. Er konnte öfters eine grünliche Verfärbung der ganzen Sonnenscheibe kurz vor ihrem Untergange beobachten. Einige Male sah er das letzte Segment grün - wie Brandt es beschreibt - und in anderen Fällen waren nur die letzten Strah"en nach dem Verschwinden der Scheibe grün - wie in meinen Beobachtungen - diese aber besonders glänzend, etwa zwei Sekunden lang. Für den Sonnenaufgang gilt nach Groff dasselbe. Einmal sah Groff den grünen Strahl, als die Sonne hinter einer Wolke verschwand, deren oberer Rand etwa $5^{\circ}$ über dem Horizont stand. Als dann die Sonne am unteren Rande der Wolke wieder hervorgekommen war (etwa $4^{\circ}$ über dem Horizont) und nun unterging, konnte er den griunen Strabl zum zweitenmal beobachten. Was die Erk arung anlangt, so scheint es mir, dab zwar besondere Bedingrungen in der Atmosphäre vorhanden sein müssen, damit die Enscheinung beobachtet werden kann, daB aber die Färbung auf physiologischen Bedingungen beruht, wie Brandt annimmt.

Am meisten Ähnlichkeit dürften die Bedingungen der Sichtbarkeit des grünen Strahls mit denen des Farbenkontrastes haben, wie sie z. B. in dem sogen. "Florversuch" verwirklicht werden. Bei diesem Ver. such induziert die Farbe des Grundes, die nur von geringer Sättigung zu sein braucht, in einem farblos bellen Felde eine komplementäre Farbenempfindung, die viel gesättigter sein kann als die Farbe des Grundes. Bei brennend rotem Abendhimmel ist die Erscheinung daher nicht zu erwarten, sondern nur bei bellrotem oder hellpurpurrotem (ungesättigtem) Abendhimmel. Eine hellsmaragdgrüne Färbung ist beim Monde unter ana logen Bedingungen zu beobachten: Ich sah einige Male den Mond kurz vor Sonnenaufgang hinter einem dünnen durchsichtigen Wolkenschleier, der durch das Morgenrot rosa erschien; schön grün gefürbt. Groff erwähnt, da $B$ er auch statt grün eine blaue Färbung gasehen habe. In Äggpten ist der Horizont durch den feinen Wüstenstaub fast stets gelb, so das blau als Komple mentärfarbe hierzu auftreten könnte, wenn durch be sóndere Umstände das Rot des Abendhimmels stark zurücktritt.

Bonn, den 15. Mai 1920.

\section{A. Pütter.}

1) William N. Groff, La plus ancienne observation d'un phenomène naturel ou astronomique. In Deurres égytologiques de William N. Groff, Paris 1908 S. $171-180$, zuerst mitgeteilt in Bulletin de l'Institut égyptien 1893, 3e série, t. IV, p. 149-156. 